\title{
JOINT SPECTRUM MANAGEMENT AND CONSTRAINED PARTIAL CROSSTALK CANCELLATION IN A MULTI-USER XDSL ENVIRONMENT
}

\author{
Jan Vangorp, Paschalis Tsiaflakis and Marc Moonen \\ Department of Electrical Engineering \\ Katholieke Universiteit Leuven, Belgium \\ \{Jan.Vangorp, Paschalis.Tsiaflakis, Marc.Moonen\}@esat.kuleuven.be
}

\author{
Jan Verlinden \\ DSL Experts Team \\ Alcatel Bell, Belgium \\ Jan.VJ.Verlinden@alcatel.be
}

\begin{abstract}
In modern DSL systems, crosstalk is a major source of performance degradation. Crosstalk cancellation techniques have been proposed to mitigate the effect of crosstalk. However, the complexity of these crosstalk cancellation techniques grows with the square of the number of lines. Therefore one has to be selective in cancelling crosstalk to reduce complexity. Secondly, crosstalk cancellation requires signal-level coordination between transmitters or receivers, which is not always available. Because of accessibility constraints, crosstalk between some lines cannot be cancelled and so has to be mitigated through spectrum management. This paper presents a solution for the joint spectrum management and constrained partial crosstalk cancellation problem. The complexity of the partial crosstalk cancellation part of the problem is reduced based on a line selection and user independence observation. However, to fully benefit from these observations, power loading has to be applied for spectrum management. We therefore consider ON/OFF power loading, which has only a minor performance degradation compared to normal power loading. The algorithm will be compared to currently available algorithms for independent spectrum management and partial crosstalk cancellation.
\end{abstract}

\section{INTRODUCTION}

Current $\mathrm{xDSL}$ access networks are evolving into mixtures of various DSL flavours. Traditional ADSL lines provisioning customers over longer distances are starting to share binders with VDSL lines deployed from remote terminals. These network topologies suffer from electromagnetic coupling resulting in crosstalk between lines. Because current xDSL systems under development use higher frequencies to meet the demand for high data rates, crosstalk is becoming particularly harmful. Moreover, significant line length variations and mixed deployments from central offices (CO's) and remote terminals (RT's) create a near-far effect in the upstream and downstream direction respectively. This causes crosstalk to sometimes overpower the direct signals. As a result, crosstalk, being 10$15 \mathrm{~dB}$ larger than the background noise, is a major limiting factor in the performance of xDSL systems.

One strategy for dealing with this crosstalk is crosstalk cancellation. Several crosstalk cancellation techniques have been proposed to remove crosstalk [1] [2] [3]. In [4] [5] it is shown that a simple linear zero-forcing canceller or linear precompensator performs near optimally in an xDSL environment.

Even for these simple linear cancellers, the complexity grows with the square of the number of lines. For example, in a binder of 8 VDSL lines transmitting on 4096 tones at a block rate of 4000

\footnotetext{
Jan Vangorp is a research assistant with the ESAT/SISTA laboratory.

Paschalis Tsiaflakis is a research assistant with the F.W.O. Vlaanderen.

This research work was carried out at the ESAT laboratory of the Katholieke Universiteit Leuven, in the frame of CELTIC/IWT project 040049: 'BANITS' Broadband Access Networks Integrated Telecommunications', the Belgian Programme on Interuniversity Attraction Poles, initiated by the Belgian Federal Science Policy Office IUAP P5/22 ('Dynamical Systems and Control: Computation, Identification and Modelling') and P5/11 ('Mobile multimedia communication systems and networks') and was partially sponsored by Alcatel-Bell.
}

blocks per second, the runtime complexity of crosstalk cancellation exceeds 1 billion multiplications per second. Because most of the crosstalk originates from a limited number of lines on a limited number of tones, a fraction of this complexity suffices to cancel most of the crosstalk. This is called partial crosstalk cancellation [6] [7].

Crosstalk cancellation requires signal-level coordination at either the transmitter or receiver, i.e. the signals transmitted on interfering lines should be known to the canceller. Oftentimes, not all interfering lines can be cancelled because their signals are not accessible. This is the case in a mixed CO-RT deployment where $\mathrm{CO}$ and RT reside in different geographical locations. Here partial crosstalk cancellation at the $\mathrm{CO}$ side has to be done independent of the partial crosstalk cancellation at the RT side. Secondly, accessibility constraints restrict the number of lines that can have signal-level coordination, even if they are at the same location. For example, crosstalk cancellation may not be possible between lines connected to different line cards.

In such situations, spectrum management can be used to mitigate the crosstalk originating from lines that are not accessible. This is a second strategy for dealing with crosstalk. Instead of cancelling the crosstalk after it has occurred, transmit spectra are chosen such that the effect of crosstalk is minimized.

Currently available algorithms independently solve the spectrum management and partial cancellation problem. A spectrum management algorithm first chooses spectra that try to avoid crosstalk. As an example, Optimal Spectrum Balancing (OSB) [8] [9] can be used to calculate optimal spectra that minimize the effect of crosstalk. Given these spectra, a partial crosstalk cancellation scheme is used to cancel the remaining crosstalk. This approach can be suboptimal. The spectrum management algorithm does not take into account that a certain amount of crosstalk can be cancelled afterwards and hence the spectra will be overly conservative.

A better solution can be obtained if the spectrum management and partial crosstalk cancellation problems are solved jointly. In [10], partial crosstalk cancellation based on resource allocation [6] is combined with Iterative Waterfilling (IW) spectrum management [11] in an iterative fashion. However, IW tends to be highly suboptimal in near-far scenarios. In this paper, the OSB algorithm is extended to include constrained partial crosstalk cancellation.

\section{JOINT SPECTRUM MANAGEMENT AND CONSTRAINED PARTIAL CROSSTALK CANCELLATION}

\subsection{System Model}

Most current DSL systems use Discrete Multi-Tone (DMT) modulation. The available frequency band is divided in a number of parallel subchannels or tones. Each tone is capable of transmitting data independently from other tones, and so the transmit power and the number of bits can be assigned individually for each tone. This gives a large flexibility in optimally shaping the transmit spectrum to minimize the effect of crosstalk.

Transmission for a binder of $N$ users can be modelled on each tone $k$ by

$$
\mathbf{y}_{k}=\mathbf{H}_{k} \mathbf{x}_{k}+\mathbf{z}_{k} \quad k=1 \ldots K .
$$

The vector $\mathbf{x}_{k}=\left[x_{k}^{1}, x_{k}^{2}, \ldots, x_{k}^{N}\right]^{T}$ contains the transmitted signals on 
tone $k$ for all $N$ users. $\left[\mathbf{H}_{k}\right]_{n, m}=h_{k}^{n, m}$ is an $N \times N$ matrix containing the channel transfer functions from transmitter $m$ to receiver $n$. The diagonal elements are the direct channels, the off-diagonal elements are the crosstalk channels. $\mathbf{z}_{k}$ is the vector of additive noise on tone $k$, containing thermal noise, alien crosstalk, RFI,... The vector $\mathbf{y}_{k}$ contains the received symbols.

To take crosstalk cancellation into account, an equivalent channel $\tilde{\mathbf{H}}$ is introduced. This is the same channel as the original channel $\mathbf{H}$, but with off-diagonal elements set to 0 where the crosstalk is cancelled. If user $n$ is cancelling crosstalk originating from user $m$ on tone $k$, then $\tilde{h}_{k}^{n, m}=0$. We refer to [6] [7] where procedures are explained for cancelling individual crosstalk channels, based on particular DSL channel characteristics (row/column-wise diagonal dominance).

We denote the transmit power as $s_{k}^{n} \triangleq \Delta_{f} E\left\{\left|x_{k}^{n}\right|^{2}\right\}$, the noise power as $\sigma_{k}^{n} \triangleq \Delta_{f} E\left\{\left|z_{k}^{n}\right|^{2}\right\}$. The vector containing the transmit power of user $n$ on all tones is $\mathbf{s}^{n} \triangleq\left[s_{1}^{n}, s_{2}^{n}, \ldots, s_{K}^{n}\right]^{T}$. The DMT symbol rate is denoted as $f_{s}$, the tone spacing as $\Delta_{f}$.

It is assumed that each modem treats interference from other modems as noise. When the number of interfering modems is large, the interference is well approximated by a Gaussian distribution. Under this assumption the achievable bit loading of user $n$ on tone $k$, given the transmit spectra of all modems in the system, is

$$
b_{k}^{n} \triangleq \log _{2}\left(1+\frac{1}{\Gamma} \frac{\left|\tilde{h}_{k}^{n, n}\right|^{2} s_{k}^{n}}{\sum_{m \neq n}\left|\tilde{h}_{k}^{n, m}\right|^{2} s_{k}^{m}+\sigma_{k}^{n}}\right),
$$

where $\Gamma$ denotes the SNR-gap to capacity, which is function of the desired BER, the coding gain and noise margin. The bitload vector for user $n$ is then $\mathbf{b}^{n}=\left[b_{1}^{n}, b_{2}^{n}, \ldots, b_{K}^{n}\right]$ and $\mathbf{b}=\left[\mathbf{b}^{1}, \mathbf{b}^{2}, \ldots, \mathbf{b}^{N}\right]$. The data rate and total power for user $n$ is

$$
R^{n}=f_{s} \sum_{k} b_{k}^{n} \quad \text { and } \quad P^{n}=\sum_{k} s_{k}^{n}
$$

\subsection{Problem Statement}

The joint spectrum management and constrained partial crosstalk cancellation problem amounts to finding an optimal allocation of transmit power and selection of the crosstalk to cancel, thereby maximizing the capacity of the network. In doing so, there are a number of constraints.

First of all, there is a total power constraint $P^{n, t o t}$ for each user. This constraint ensures the user's total power does not exceed the maximum allowed total transmit power. On top of this constraint there can be a spectral mask constraint $s_{k}^{n, \text { mask }}$ for each tone to guarantee electromagnetic compatibility with other systems.

Secondly, because of the runtime complexity of full crosstalk cancellation, there is a limited amount of resources for crosstalk cancellation. The cancellation of the crosstalk from one crosstalker on a tone is done by one cancellation tap [6] [7]. The number of cancellation taps that can be used is constrained by the cancellation tap constraint $C^{\text {tot }}$ [12]. Furthermore, in a bundle of lines, not all crosstalk can be cancelled. This is the case when receivers are in different geographical locations or when lines are terminating on different line cards. These scenarios can be modelled by multiple cancellation tap constraints $C^{q, t o t}$, each constraint for a subset $q$ of lines with full signal-level access.

Finally, there is a rate constraint $R^{n, \text { target }}$ for each user. Typically, service providers offer a number of profiles to guarantee a certain Quality of Service. The rate constraint then indicates a minimum data rate required by the user.

Joint spectrum management and constrained partial crosstalk cancellation then results in solving the following maximization problem, adopted from [12] and extended to include accessibility constraints:

$$
\begin{aligned}
& \max _{\mathbf{s}, \mathbf{c}} \sum_{n=1}^{N} R^{n} \\
& \text { s.t. } \quad P^{n} \leq P^{n, t o t} \\
& 0 \leq s_{k}^{n} \leq s_{k}^{n \text { mask }} \\
& \begin{array}{ll}
C^{q}=\sum_{k=1}^{K} \sum_{m \in i^{q}} \sum_{n \in i^{q}} c_{k}^{n, m} \leq C^{q, t o t} & \\
R^{n} \geq R^{n, \text { target }} & \\
& n=1 \ldots Q
\end{array} \\
& \text { with } \quad\left[\mathbf{c}_{k}\right]_{n, m}=c_{k}^{n, m} \quad c_{k}^{n, m}=\left\{\begin{array}{lll}
0 & \Rightarrow & \tilde{h}_{k}^{n, m}=h_{k}^{n, m} \\
1 & \Rightarrow & \tilde{h}_{k}^{n, m}=0
\end{array}\right.
\end{aligned}
$$

where $\mathbf{s}=\left[\mathbf{s}^{1}, \mathbf{s}^{2}, \ldots, \mathbf{s}^{N}\right]$ and $\mathbf{c}=\left[\mathbf{c}_{1}, \mathbf{c}_{2}, \ldots, \mathbf{c}_{K}\right] . \mathbf{c}_{k}$ is a matrix containing the crosstalk cancellation configuration for tone $k$. $c_{k}^{n, m}=1$ indicates that a cancellation tap is assigned on tone $k$ for cancelling crosstalk on line $n$ originating from line $m$. Because of accessibility constraints, $n$ and $m$ are restricted to the subset of line indices $i^{q}$ which have full signal-level control. For lines $n, m$ that have no signal-level control, $c_{k}^{n, m}=0, \forall k$.

\subsection{Dual Decomposition}

Optimization problem (2) is a non-convex problem. To find the global optimum one has to exhaustively search through all possible transmit spectra $\mathbf{s}$ and cancellation tap configurations c. Because some constraints are coupled over the tones, this results in an exponential complexity in the number of tones. By using a dual decomposition the complexity can be made linear [8] [9] [12]. This is done by using Lagrange multipliers to move the constraints coupled over tones into the objective function of the optimization problem:

$$
\begin{aligned}
& \mathbf{s}^{o p t}, \mathbf{c}^{o p t}=\operatorname{argmax}_{\mathbf{s}, \mathbf{c}} \sum_{n=1}^{N} \omega_{n} R^{n}+\sum_{n=1}^{N} \lambda_{n}\left(P^{n, t o t}-\sum_{k=1}^{K} s_{k}^{n}\right) \\
& +\sum_{q=1}^{Q} v_{q}\left(C^{q, t o t}-\sum_{k=1}^{K} \sum_{n \in i^{q}} \sum_{m \in i^{q}} c_{k}^{n, m}\right) \\
& \text { subject to } \quad 0 \leq s_{k}^{n} \leq s_{k}^{n, \text { mask }} \quad n=1 \ldots N \\
& \lambda_{n} \geq 0, \omega_{n} \geq 0 \quad n=1 \ldots N \\
& v_{q} \geq 0 \quad q=1 \ldots Q
\end{aligned}
$$

where $\omega_{n}, \lambda_{n}$ and $v_{q}$ are Lagrange multipliers. For a given set of $\boldsymbol{\omega}=\left[\omega_{1}, \ldots, \omega_{N}\right]^{T}, \boldsymbol{\lambda}=\left[\lambda_{1}, \ldots, \lambda_{N}\right]^{T}$ and $\boldsymbol{v}=\left[v_{1}, \ldots, v_{Q}\right]^{T}$, the optimization problem can then be solved in a per-tone fashion:

$$
\begin{gathered}
\text { for } k=1 \ldots K, \quad \mathbf{s}_{k}^{o p t}, \mathbf{c}_{k}^{\text {opt }}=\operatorname{argmax} \\
-\sum_{n=1}^{N} \lambda_{n} s_{k}^{n}-\sum_{q=1}^{Q} \sum_{n \in i^{q}} \sum_{m \in i^{q}} v_{q} c_{k}^{n, m}
\end{gathered}
$$

Maximization of (4) for given Lagrange multipliers can be performed by an exhaustive search. For each tone, the objective function should be evaluated for all possible combinations of the transmit power levels and cancellation tap configurations of the users. The combination giving the largest value for this expression is the optimal allocation of transmit power and cancellation taps for this tone. Instead of power loading, one could also perform bit loading by choosing all possible bit allocations. This would be the case when (2) would be reformulated as an optimization problem in $\{\mathbf{b}, \mathbf{c}\}$ instead of $\{\mathbf{s}, \mathbf{c}\}$.

After the optimization, the constraints can be checked. By choosing appropriate values for the Lagrange multipliers, the constraints can be enforced. $\boldsymbol{\lambda}$ and $\boldsymbol{v}$ can be seen as a cost for power and crosstalk cancellation taps respectively. Larger values for these Lagrange multipliers result in less power and allocated cancellation taps. The data rates of the users are weighted by $\boldsymbol{\omega}$, thereby giving a level of importance to the users. In this way, all possible trade offs can be made to enforce the data rate constraints. For given $\boldsymbol{\omega}, \boldsymbol{\lambda}$ and $\boldsymbol{v}$, the constraints can be checked by performing an exhaustive search for all tones. 
To solve (2) by (4), $\boldsymbol{\omega}, \boldsymbol{\lambda}$ and $\boldsymbol{v}$ should be tuned to enforce the constraints. In [8], an efficient Lagrange multiplier search procedure for $\boldsymbol{\omega}$ and $\boldsymbol{\lambda}$ is presented. This procedure can be easily extended to include $\boldsymbol{v}$, resulting in the following update formula for the Lagrange multipliers:

$$
\begin{gathered}
{\left[\begin{array}{c}
\Delta \boldsymbol{\omega} \\
\Delta \boldsymbol{\lambda} \\
\Delta \boldsymbol{v}
\end{array}\right]=-\mu\left[\begin{array}{l}
\mathbf{R}-\mathbf{R}^{\text {target }} \\
\mathbf{P}^{\text {tot }}-\mathbf{P} \\
\mathbf{C}^{\text {tot }}-\mathbf{C}
\end{array}\right] \Rightarrow} \\
{\left[\begin{array}{l}
\boldsymbol{\omega} \\
\boldsymbol{\lambda} \\
\boldsymbol{v}
\end{array}\right]^{t+1}=\left(\left[\begin{array}{l}
\boldsymbol{\omega} \\
\boldsymbol{\lambda} \\
\boldsymbol{v}
\end{array}\right]^{t}-\mu\left[\begin{array}{l}
\mathbf{R}-\mathbf{R}^{\text {target }} \\
\mathbf{P}^{\text {tot }}-\mathbf{P} \\
\mathbf{C}^{t o t}-\mathbf{C}
\end{array}\right]\right)}
\end{gathered}
$$

where $(x)^{+}$means $\max (0, x)$ and where $\mathbf{R}=\left[R^{1}, \ldots, R^{N}\right]^{T}, \mathbf{P}=$ $\left[P^{1}, \ldots, P^{N}\right]^{T}$ and $\mathbf{C}=\left[C^{1}, \ldots, C^{Q}\right]^{T}$ are vectors with the total powers, data rates and number of cancellation taps corresponding to the Lagrange multipliers at hand. This update formula is used in algorithm 1 adopted from [8].

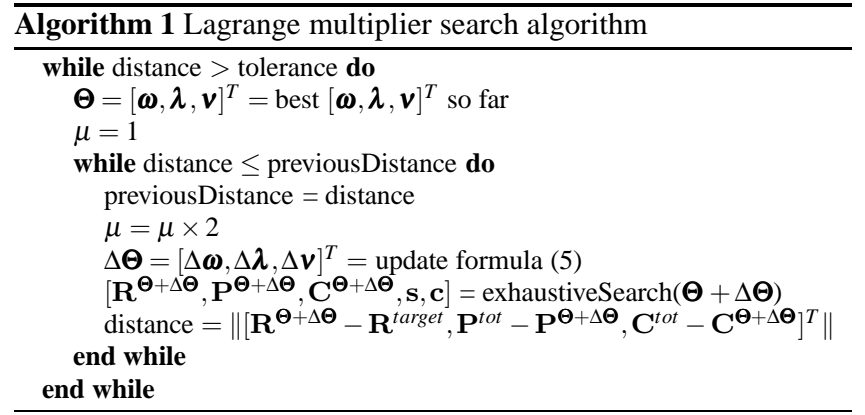

Note that all the Lagrange multipliers are updated in parallel. In [8] it is shown that adding extra Lagrange multipliers does not increase the number of steps required for convergence. The search procedure typically converges in 50 to 150 steps. Therefore, the cancellation tap constraint only adds to the complexity of the per-tone exhaustive search.

\subsection{Complexity}

The joint spectrum management and constrained partial crosstalk cancellation problem (2) is a non-convex constrained optimization problem. Without the dual decomposition, finding the global optimum requires an exhaustive search over all possible solutions. First, assume there are no accessibility constraints, so all crosstalk can be cancelled. On a certain tone, a user has to decide which crosstalk of $N-1$ other users has to be cancelled. There are $2^{N-1}$ possibilities to do this. Together with $B$ possibilities for bit or power loading, this results in a total of $B 2^{N-1}$ possibilities for each user on each tone and hence a total complexity of $\mathscr{O}\left(\left(B 2^{N-1}\right)^{K N}\right)$.

The dual decomposition decouples the problem over the tones, therefore reducing the exponential complexity in the number of tones $K$ to linear complexity: $\mathscr{O}\left(K\left(B 2^{N-1}\right)^{N}\right)$. This amounts to $K$ exhaustive searches of complexity $\mathscr{O}\left(\left(B 2^{N-1}\right)^{N}\right)$. This is an enormous reduction in complexity. However, this solution is still computationally intractable because of the remaining complexity of the per-tone exhaustive search, which is $\left(2^{N-1}\right)^{N}$ times more complex than solving the spectrum management problem without crosstalk cancellation. In a 4-user upstream VDSL scenario for example, it takes 20 days to calculate optimal spectra with OSB on a Pentium IV. Adding partial crosstalk cancellation to the problem would then take about 225 years.

The dual decomposition approach is only feasible if the pertone exhaustive search can be performed with manageable complexity, which was also concluded in [12]. In the next section, methods are introduced to make this possible.

\section{COMPLEXITY REDUCTION}

The complexity of the per-tone exhaustive search for the joint spectrum management and constrained partial crosstalk cancellation problem is $\mathscr{O}\left(K\left(B 2^{N-1}\right)^{N}\right)$ in the case where all crosstalk can be cancelled (section 2.4). This can be rewritten as $\mathscr{O}\left(K B^{N}\left(2^{N-1}\right)^{N}\right)$, clearly showing the per-tone complexity due to spectrum management, $\mathscr{O}\left(B^{N}\right)$, and partial crosstalk cancellation, $\mathscr{O}\left(\left(2^{N-1}\right)^{N}\right)$. In this section we focus on reducing the complexity originating from these two individual subproblems.

\subsection{Partial Crosstalk Cancellation}

This subsection again starts with the assumption that there are no accessibility constraints, i.e. that all crosstalk can be cancelled. Later, observations will be extended to the case when there are multiple line cards which cannot access each others lines for crosstalk cancellation.

To determine the optimal allocation of crosstalk cancellation taps for a given bit or power loading on a certain tone, all of the $\left(2^{N-1}\right)^{N} \approx 2^{N^{2}}$ possible allocations have to be evaluated. Even for a limited number of users this is already too large. Fortunately, many of these possibilities can be eliminated based on two observations: line selection and user independence.

- Line Selection: From (1) it can be seen that to maximize the capacity, one should allocate crosstalk cancellation taps to cancel the users that are causing the largest crosstalk. Therefore, if $r$ crosstalk cancellation taps are available, they should be used to cancel the $r$ largest sources of crosstalk.

As a consequence, instead of $2^{N-1}$ possibilities, there are now only $N$ possibilities: cancel no crosstalker, cancel the strongest crosstalker, cancel the 2 strongest crosstalkers, ..., cancel the $N-1$ strongest crosstalkers.

- User Independence: From (1) it can be seen that if user $n$ allocates a crosstalk cancellation tap to cancel crosstalk caused by user $m\left(\right.$ e.g. $\left.\tilde{h}_{k}^{n, m}=0\right)$ this only has an influence on the capacity of user $n$. Therefore, when power loading is applied, the users are decoupled so they can choose a crosstalk cancellation configuration independently.

As a consequence, the exponential complexity in $N$ is reduced to linear complexity. Instead of one big search over all users, there are $N$ independent searches for the users. This observation, together with line selection, results in the following complexity reduction:

$$
\left(2^{N-1}\right)^{N} \quad \stackrel{\text { line selection }}{\longrightarrow} N^{N} \quad \text { user independence } \quad N N
$$

It is noted that in the case of optimal bit loading, user independence does not hold. Adding a crosstalk cancellation tap changes the power needed to transmit a certain number of bits, thus also the crosstalk to other users changes. This may affect the configuration of crosstalk cancellation taps for these other users.

These observations can be easily extended to the case where there are multiple line cards. In this case, there are accessibility constraints, reducing the number of crosstalkers that can be cancelled. Assume there are $Q$ line cards. Line card $q$ has access to $M_{q}$ lines, with $\sum_{q=1}^{Q} M_{q}=N$. The complexity reduction by line selection and user independence is then summarized in table $\mathbf{1}$.

In a 8-user case, the observations reduce the number of crosstalk cancellation configurations from $2^{56}$ to $2^{6}$. If there are 2 line cards, each having 4 lines, the number of crosstalk cancellation configurations is reduced from $2^{24}$ to $2^{5}$.

Note that despite drastic complexity reductions, the solution is still optimal.

\subsection{Spectrum Management: ON/OFF Power Loading}

In this subsection, the complexity of the spectrum management part of the problem is reduced. Despite the complexity reduction pro- 
Table 1: Complexity reduction partial crosstalk cancellation

\begin{tabular}{|c|c|c|}
\hline & 1 line card & $Q$ line cards \\
\hline full complexity & $\mathscr{O}\left(\left(2^{N-1}\right)^{N}\right)$ & $\mathscr{O}\left(\prod_{q=1}^{Q}\left(2^{M_{q}-1}\right)^{M_{q}}\right)$ \\
\hline line selection & $\mathscr{O}\left((N)^{N}\right)$ & $\mathscr{O}\left(\prod_{q=1}^{Q}\left(M_{q}\right)^{M_{q}}\right)$ \\
\hline user independence & $\mathscr{O}\left(N 2^{N-1}\right)$ & $\mathscr{O}\left(\sum_{q=1}^{Q} M_{q} 2^{M_{q}-1}\right)$ \\
\hline line selection \& user independence & $\mathscr{O}(N N)$ & $\mathscr{O}\left(\sum_{q=1}^{Q} M_{q} M_{q}\right)$ \\
\hline
\end{tabular}

vided by dual decomposition, OSB is still too complex for scenarios with more than 3 users. The reason is the per-tone exhaustive search which still has exponential complexity in the number of users: $\mathscr{O}\left(B^{N}\right)$. In [13] [14] an iterative procedure is used to make this complexity linear. However, optimality cannot be guaranteed.

In this paper, the complexity is combated by reducing $B$, the number of possible transmit levels (for power loading) or bit allocations (for bit loading). Originally, for OSB, typical values for $B$ are 60 in the case of power loading and 14 in the case of bit loading. Therefore, bit loading would be the most efficient method for OSB However, as shown in the previous subsection, power loading is necessary to fully benefit from the line selection and user independence observations when also deciding on the partial crosstalk cancellation configuration. By limiting the transmit spectra to ON/OFF power loading, $B=2$, the complexity is reduced from $\mathscr{O}\left(B^{N}\right)$ to $\mathscr{O}\left(2^{N}\right)$. This ON/OFF power loading problem equals (4) with the spectral mask constraints replaced by

$$
s_{k}^{n} \in\left\{0, s_{k}^{n, O N}\right\} \text { with } s^{n, O N} \leq s_{k}^{n, \text { mask }} .
$$

This ON/OFF power loading results in simple transmit spectra, similar to what is used in current ADSL systems.

To define the ON-level $s^{n, O N}$ for each user, the algorithm described in [15] can be used. It is shown there that the extra constraint of ON/OFF spectra only results in a small performance degradation of $10-15 \%$ compared to the full OSB algorithm.

When combining the line selection and user independence observations with ON/OFF power loading, the optimization of (2) can be done in a matter of minutes instead of the original 225 years.

\section{SIMULATION RESULTS}

In this section the performance is analyzed when solving the joint spectrum management and constrained partial crosstalk cancellation problem as opposed to independently solving these two problems. An upstream VDSL scenario is considered as shown in figure 1, with full signal-level coordination. A line diameter of $0.5 \mathrm{~mm}$ (24 AWG) is used and the maximum transmit power is $11.5 \mathrm{dBm}$. The SNR gap $\Gamma$ is set to $12.9 \mathrm{~dB}$, corresponding to a target symbol error probability of $10^{-7}$, coding gain of $3 \mathrm{~dB}$ and a noise margin of $6 \mathrm{~dB}$. The tone spacing is $\Delta_{f}=4.3125 \mathrm{kHz}$ and the DMT symbol rate $f_{s}=4 \mathrm{kHz}$

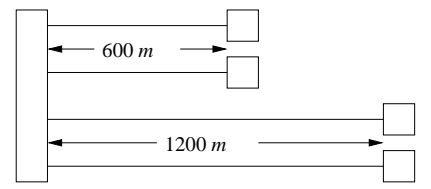

Figure 1: 4-user VDSL scenario

In figure 2 rate regions are shown to compare the performance of the joint solution and the independent solution obtained by independently solving the spectrum management problem (with ON/OFF loading) and the partial cancellation problem. The rate regions show significant performance gains of the joint solution over the independent solution. Because the independent solution

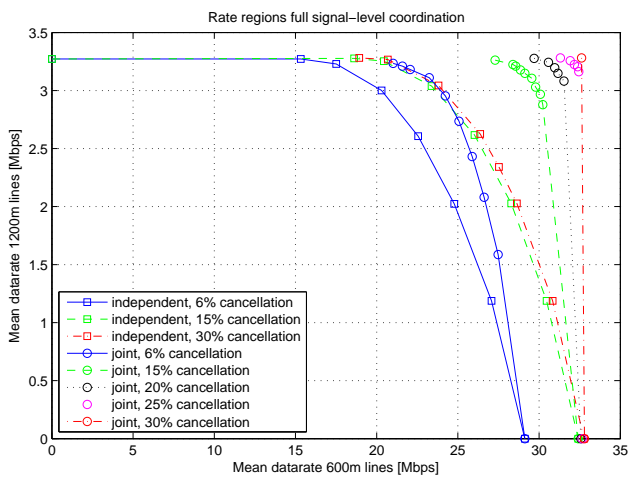

Figure 2: Rate regions 4-user VDSL scenario

first independently solves the spectrum management problem, the transmit spectra are chosen to avoid crosstalk. This can be seen in figure 3(a)(c), where the PSD is shown along with the allocation of cancellation taps for each user and the originating user of the crosstalk that is cancelled. For this strong crosstalk scenario the transmit spectra result in long and short lines occupying different frequency bands. When the partial crosstalk cancellation problem is solved, there is not much crosstalk left to cancel. Therefore, only a limited crosstalk cancellation tap budget can be used effectively. Figure 2 shows that no performance is gained by increasing the crosstalk cancellation tap budget beyond $15 \%$ of full cancellation.

When the spectrum management problem and the partial crosstalk cancellation problem are solved jointly, transmit spectra are chosen such that only crosstalk that cannot be cancelled is avoided. This can be seen in figure $3(\mathbf{b})(\mathbf{d})$, where all crosstalk cancellation taps can now be used effectively. Depending on the crosstalk cancellation tap budget, transmit spectra can overlap on frequencies with the highest capacity, resulting in significant performance gains.

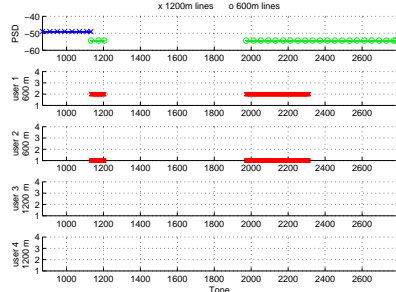

(a)

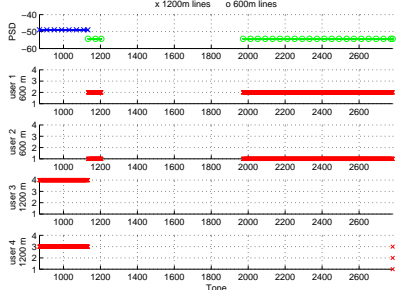

(c)

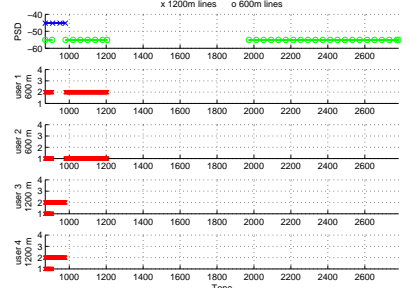

(b)

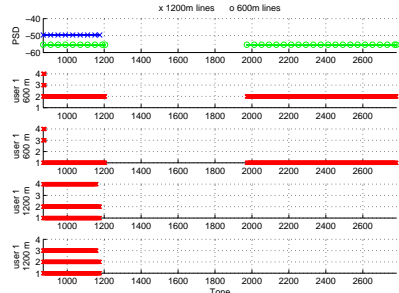

(d)
Figure 3: Spectra and cancellation configurations: (a) independent solution, $6 \%$ of full cancellation; (b) joint solution, $6 \%$ of full cancellation; (c) independent solution, 30\% of full cancellation; (d) joint solution, $30 \%$ of full cancellation;

When there are restrictions on the signal-level coordination, a choice has to be made as to which lines will be connected to the same line card. In this case, there are two possibilities: connect the 


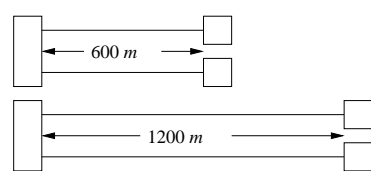

(a)

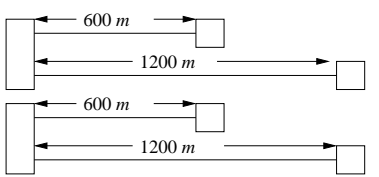

(b)
Figure 4: 4-user VDSL scenarios with limited signal-level coordination

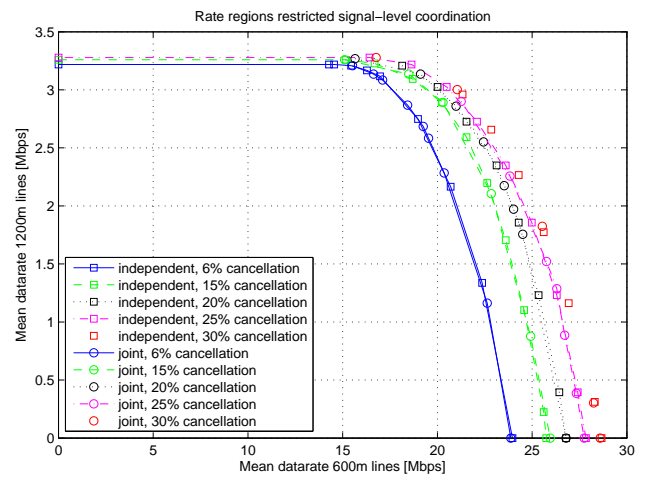

Figure 5: Rate regions 4-user VDSL scenario with limited signallevel coordination: equal line lengths together

lines with the same length to the same line card (figure 4(a)) or connect lines with different length to the same line card (figure 4(b)).

Connecting lines with the same length to the same line card results in the rate regions of figure 5. Only limited performance is gained by increasing the crosstalk cancellation tap budget. This is caused by the fact that the long lines do not have access to the short lines. Therefore, this major source of crosstalk cannot be cancelled. As a result, the spectrum management has to be used to avoid this crosstalk and both groups of lines occupy different frequency bands. Therefore the joint and independent solutions are similar and only a limited number of crosstalk cancellation taps can be used effectively.

When lines of different length are connected to the same line card, the rate regions of figure $\mathbf{6}$ are obtained. Again, there is no significant difference between the joint and independent solutions. Because the long lines cannot access all short lines, there will be severe crosstalk that cannot be cancelled if these lines would use the same frequency band. As a consequence, long and short lines use different frequency bands. Moreover, because lines of equal length are on different line cards, no crosstalk cancellation taps can be assigned.

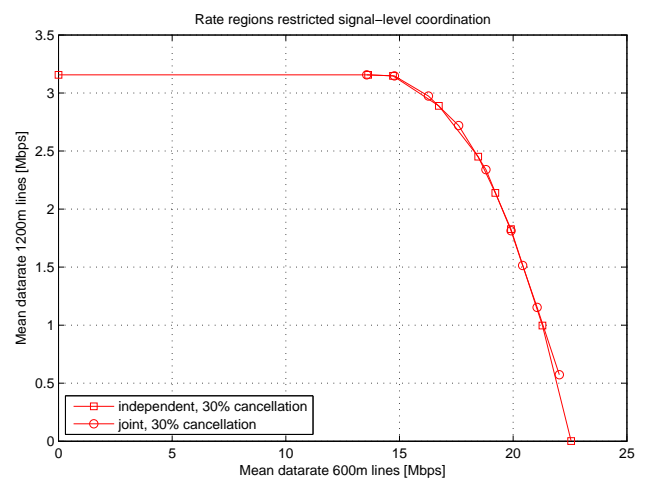

Figure 6: Rate regions 4-user VDSL scenario with limited signallevel coordination: mixed line lengths

\section{CONCLUSION}

In this paper, a solution was presented to jointly solve the spectrum management and constrained partial crosstalk cancellation problem based on a dual decomposition approach. The complexity of the partial crosstalk cancellation part of the solution was reduced to a minimum based on a line selection and a user independence observation. However, to fully benefit from these observations, power loading has to be applied for spectrum management. We have therefore considered ON/OFF power loading, which only has a minor performance degradation compared to the original power loading.

It was shown that when the spectrum management problem and partial crosstalk cancellation problem are solved independently, only a limited number of crosstalk cancellation taps can be used effectively because crosstalk is avoided in the first place by the spectrum management. When jointly solving the problems, only crosstalk that cannot be cancelled is avoided, thereby significantly increasing performance.

\section{REFERENCES}

[1] G. Taubock and W. Henkel, "MIMO systems in the subscriber-line network," in 5th Int. OFDM Workshop, Sep. 2000, pp. 18.1-18.3.

[2] G. Ginis and J. M. Cioffi, "Vectored Transmission for Digital Subscriber Line Systems," IEEE J. Sel. Area. Comm., vol. 20, no. 5, pp. 1085-1104, Jun. 2002.

[3] W. Yu and J. M. Cioffi, "Multiuser detection in vector multiple access channels using generalized decision feedback equalization," in 5th Int. Conf. Signal Processing, Aug. 2000.

[4] R. Cendrillon, G. Ginis, E. Van den Bogaert and M. Moonen, "A Near-optimal Linear Crosstalk Canceler for VDSL," accepted for IEEE Trans. Sign. Proc., 2004. [Online]. Available: http://homes.esat.kuleuven.be/ sistawww/cgi-bin/pub.pl

[5] R. Cendrillon, G. Ginis, E. Van den Bogaert and M. Moonen, "A Near-optimal Linear Crosstalk Precoder for VDSL," accepted for IEEE Trans. Comm., 2004. [Online]. Available: http://homes.esat.kuleuven.be/ sistawww/cgi-bin/pub.pl

[6] R. Cendrillon, M. Moonen, G. Ginis, K. Van Acker, T. Bostoen, P. Vandaele, "Partial Crosstalk Cancellation for Upstream VDSL," EURASIP J. Applied Sig. Proc., vol. 2004, no. 10, pp. 1520-1535, Aug. 2004.

[7] R. Cendrillon, M. Moonen, G. Ginis, K. Van Acker, T. Bostoen and P. Vandaele, "Partial Crosstalk Precompensation for Downstream VDSL,” Elsevier Signal Processing, pp. 2005-2019, Nov. 2004.

[8] P. Tsiaflakis, J. Vangorp, M. Moonen, J. Verlinden, K. Van Acker, "An efficient Lagrange Multiplier search algorithm for Optimal Spectrum Balancing in crosstalk dominated xDSL systems," in accepted for IEEE ICASSP, 2006.

[9] R. Cendrillon, W. Yu, M. Moonen, J. Verlinden and T. Bostoen, "Optimal Multi-user Spectrum Management for Digital Subscriber Lines," accepted for IEEE Trans. Comm. [Online]. Available: http://homes.esat.kuleuven.be/ sistawww/cgi-bin/pub.pl

[10] H. Bagheri, H. Emami, M. R. Pakravan, "Iterative Joint Power Control and Partial Crosstalk Cancellation in Upstream VDSL," Int. Journal on Signal Processing, vol. 1, no. 3, pp. 195-198, 2004.

[11] W. Yu, G. Ginis, J. Cioffi, "Distributed Multiuser Power Control for Digital Subscriber Lines," IEEE J. Sel. Area. Comm., vol. 20, no. 5, pp. 1105-1115, Jun. 2002.

[12] W. Yu, R. Lui and R. Cendrillon, "Dual Optimization Methods for Multiuser Orthogonal Frequency-Division Multiplex Systems," in IEEE Globecom, vol. 1, Dec. 2004, pp. 225-229.

[13] R. Lui and W. Yu, "Low-Complexity Near-Optimal Spectrum Balancing for Digital Subscriber Lines," in IEEE ICC, May 2005.

[14] R. Cendrillon, M. Moonen, "Iterative Spectrum Balancing for Digital Subscriber Lines," in IEEE ICC, May 2005.

[15] J. Vangorp, P. Tsiaflakis, M. Moonen, J. Verlinden, K. Van Acker, “Optimal Spectrum Balancing in multi-user xDSL systems with ON/OFF power loading," in accepted for IEEE ICASSP, 2006. 